\title{
VARIAÇÃO DO TEOR DE NITROGÊNIO EM RAMOS PRODUTIVOS DE CAQUIZEIRO ${ }^{1}$
}

\author{
SINVAL XAVIER DE AGUIAR², NAGIB JORGE MELÉM JÚNIOR², \\ INÊS CRISTINA DE BATISTA FONSECA ${ }^{4}$, ANDRÉ KIKUCHI BAGATIN 5 , \\ HIDEAKI WILSON TAKAHASHI ${ }^{6}$
}

RESUMO- O objetivo deste trabalho foi avaliar a resposta do caquizeiro (Diospyros kaki L.) à adubação nitrogenada. O experimento foi conduzido em um pomar comercial no município de Faxinal, Estado do Paraná, nas coordenadas geográficas S $23^{\circ}$ 57’ 35”, W51 13’34”, altitude de 999 metros. A cultivar estudada foi "Giombo". Utilizou-se de 9 tratamentos, sendo uma testemunha sem aplicação de nitrogênio (N), e os demais resultante das épocas de aplicação de N (Maturação fisiológica dos frutos, início de poda, final de florescimento e em 15-12-06), combinadas com duas doses de N (80 e $160 \mathrm{~kg} / \mathrm{ha}$ ). O delineamento experimental foi o de blocos casualizados e 4 repetições. A parcela experimental foi constituída de 3 plantas, sendo a planta central usada para avaliação. A cada quarenta e cinco dias após o início da adubação, foram retirados aleatoriamente de cada tratamento oito ramos produtivos em diferentes pontos da planta. Na folha, os tratamentos IP e MF apresentaram os maiores teores de $\mathrm{N}$ na primavera e no verão, e uma diminuição significativa na fase final do ciclo antes da senescência e queda.Na folha e no ramo, todos os tratamentos resultaram em teor de $\mathrm{N}$ semelhante no final do ciclo.A época de aplicação, início de poda, foi a época que demonstrou menor variação de $\mathrm{N}$, independentemente da dose.

Termos para indexação:Diospyros kaki L., Adubação nitrogenada, época de aplicação de N, frutas temperadas.

\section{VARIATION OF THE NITROGEN CONTEN IN PRODUCTIVE BRANCHES OF PERSIMMON}

\begin{abstract}
The objective was to evaluate the variation of nitrogen content in productive branches of persimmon (Diospyros kaki L.) to nitrogen fertilization. The experiment was conducted in a commercial orchard in the municipality of Faxinal, state of Paraná, in geographical coordinates S $23^{\circ} 57$ ' 35 ”, W51 ' 13 ' 34 ”', altitude of 999 meters. The cultivar studied was "Giombo. It was used nine treatments, one as control without application of nitrogen $(\mathrm{N})$, and the remainders resulting from the period of $\mathrm{N}$ application ( physiological maturity of the fruits, pruning, end of flowering and on 12/15/2006) combined with two doses of $N$ (80 and $160 \mathrm{~kg} / \mathrm{ha}$ ). The experimental design was randomized blocks and 4 replications. The experimental plot was composed of 3 plants being the central plant used for assessment. Every forty-five days after fertilization were randomly taken from each treatment eight productive branches in different parts of the plant.. The leaf's treatment IP and MF showed the highest concentration of $\mathrm{N}$ in the spring and summer and one decrease in the end of the cycle before of the leaf fall and in the branch of all treatments resulted in similar levels of $\mathrm{N}$ in the end o cycle. The time of pruning the top resulted in the smallest levels of $\mathrm{N}$ in both application of $\mathrm{N}$. Index terms: Diospyros kaki L., Nitrogen fertilization , Time of application of N, Temperate Fruits.
\end{abstract}

\footnotetext{
1(Trabalho 007-09). Recebido em: 05-01-2009. Aceito para publicação em: 06-01-2010.

${ }^{2}$ Eng. Agro ${ }^{\circ}$. Mestre em Agronomia Universidade Estadual de Londrina - UEL, Departamento de Agronomia, C. P. 6001- CEP 86051990 - Londrina-PR. E-mail: sinvalxavier@yahoo.com.br;

${ }^{3}$ Eng. Agro ${ }^{\circ}$. Pesquisador da Embrapa Amapá, Doutorando Área de Agronomia. Universidade Estadual de Londrina - UEL, Departamento de Agronomia, C. P. 6001- CEP 86051-990 - Londrina-PR. E-mail: nagibmelem@gmail.com

${ }^{4}$ Estudante de Agronomia; Universidade Estadual de Londrina-UEL, Departamento de Agronomia, C. P. 6001- CEP 86051-990Londrina-PR. E-mail:ankbagatin@hotmail.com

${ }^{5}$ Eng $^{\text {. Agro }}$. Dr ${ }^{\text {a }}$, Professora Adjunto, Universidade Estadual de Londrina-UEL, Departamento de Agronomia, C. P. 6001- CEP 86051990 - Londrina-PR. E-mail: inescbf@uel.br.

${ }^{6}$ Eng. Agro $^{\circ}$. Dro ${ }^{\circ}$, Professor, Universidade Estadual de Londrina-UEL, Departamento de Agronomia, C. P. 6001- CEP 86051-990Londrina-PR. E-mail: hwilson@uel.br.
} 


\section{INTRODUÇÃO}

O caquizeiro (Diospyros kaki L.) tem como centro de origem as montanhas centrais da China, país onde é cultivado há milhares de anos (Martins; Pereira, 1989).

É uma fruteira que se adapta bem ao clima subtropical e temperado e, provavelmente, chegou ao Brasil ao final do século XIX (Park et al., 2004).

A produção mundial de frutos de caqui, em 2004, foi de 2,5 milhões de toneladas. O Brasil ocupa o quarto lugar no ranking dos produtores mundiais com produção de 162.288 toneladas, com área plantada, em 2007, de 1.570 ha, sendo o Estado do Paraná o terceiro maior produtor, com 22.494 toneladas, ficando atrás de São Paulo, com 80.101, e do Rio Grande do Sul, com 27.119 toneladas (IBGE, 2009).

Vários fatores influenciam na produção, qualidade dos frutos e incidência de doenças do caquizeiro. Dentre elas, a disponibilidade de nitrogênio (N) no solo é um fator importante e de fácil manejo. Também é de notório conhecimento que o $\mathrm{N}$ é o principal macronutriente para as plantas superiores. Segundo Epstein (2005) e Marschner (1995), o nitrogênio (N) está relacionado com a fotossíntese, respiração, desenvolvimento e atividade das raízes, absorção iônica de outros nutrientes, crescimento e diferenciação celular. A remobilização do nitrogênio nas plantas de clima temperado é um processo que se caracteriza pela sua estocagem do $\mathrm{N}$ durante o inverno, nas raízes, e sua translocação via xilema na primavera. É utilizado para o crescimento das folhas e inflorescência (Millard, 1996). De acordo com Muñoz et al. (1993), esse processo depende do $\mathrm{N}$ estocado nas raízes.

Na cultura do caquizeiro, na Itália, Bellini (2006) verificou que a absorção de nitrogênio aumenta progressivamente até o verão e depois diminui rapidamente nas estações seguintes. Verificou também que o período de consumo máximo do $\mathrm{N}$ ocorre na primavera e no verão, representando $68 \%$ do consumo total anual. Ainda segundo o mesmo autor, as necessidades nutricionais do caquizeiro variam de acordo com os diferentes períodos do ciclo da planta, sobretudo em função das fases vegetativas e reprodutivas, como: brotação, crescimento dos brotos e das folhas, florescimento, frutificação, indução da floração, fixação do fruto, desenvolvimento do fruto, amadurecimento, colheita, acumulação das reservas e entrada da dormência (Gasanov, 1984; Tanaka; Aoki, 1969, citado por George et al., 2003).

Em experimentos conduzidos na Nova Zelândia com o caquizeiro da cv "Fuyu", Clark e Smith (1986) verificaram que o teor de N na folha diminui progressivamente ao longo da estação de crescimento, em consequência do efeito da taxa de crescimento da folha, que é muito mais intenso do que a absorção dos nutrientes.

Para Choi et al. (2003), a adubação com nitrogênio no caquizeiro na estação do outono, tem melhorado o aspecto das folhas e o acúmulo de reservas de amido para a estação de florescimento, além de evitar a queda de frutos. De acordo com Gasanov (1984), quantidades adequadas de nitrogênio no caquizeiro reduzem a queda de flores e de frutos, elevando acentuadamente a produção.

Não existem trabalhos com caqui em relação à época de aplicação nem recomendações para aplicação de nitrogênio.

O objetivo do trabalho foi avaliar a variação do teor de nitrogênio em ramos produtivos de caquizeiro, à adubação nitrogenada aplicada em diferentes estádios de desenvolvimento.

\section{MATERIAL E MÉTODOS}

O experimento foi conduzido em um pomar comercial, sem irrigação, da Fazenda Bela Vista, situado no município de Faxinal, estado do Paraná, nas coordenadas geográficas S $23^{\circ} 57^{\prime} 35^{\prime \prime}$, W $51^{\circ}$ 13’34”, altitude de 999 metros. A região apresenta o tipo climático Cfa, na classificação de Köeppen, como clima subtropical. A precipitação média anual é de 1.400 a $1.600 \mathrm{~mm}$, com evapotranspiração potencial de 1.300 a $1.400 \mathrm{~mm}$. A temperatura média é de 20 a $21^{\circ} \mathrm{C}$ (Viglione et al., 2000). O solo do local do experimento foi classificado como Latossolo Vermelho distroférrico (EMBRAPA, 2006). A cultivar estudada foi a cV. “Giombo", em uma área de um hectare, cultivada no espaçamento de 3 metros entre plantas e 14 metros entre linha, com nove anos de idade. Cada parcela foi composta de 3 plantas, sendo utilizadas duas plantas como bordadura na linha e uma central para avaliação.

Foram retiradas na projeção da copa amostras de solo em duas camadas (0-20 e 20-40 cm profundidades) para análise química (Tabela 1).

Os 9 tratamentos são resultantes da combinação de 4 épocas de aplicação e duas doses de $\mathrm{N}$ mais a testemunha sem aplicação de N. A primeira época de aplicação foi no período de maturação fisiológica dos frutos (MF), caracterizado pela cor levemente amarelada na parte apical do fruto e que ocorreu em 20-03-2006; a segunda aplicação de $\mathrm{N}$ foi no início da poda (IP), dia 01-07-2006, a terceira aplicação foi no final do florescimento (FF), dia 20-10-2006 e a quarta aplicação no dia 15-12-2006. O delineamento experimental foi o de blocos casualizados e 4 
repetições. O adubo utilizado foi o nitrato de amônio, aplicado em cobertura, na projeção da copa, em duas doses: 80 e $160 \mathrm{~kg}$ de $\mathrm{N} \mathrm{ha}^{-1}$.

Foram retirados oito ramos com brotações emitidas no ano do experimento com frutos e folhas, em diferentes pontos da árvore,a cada 45 dias após o início dos tratamentos, nos seguintes pontos: 6 ramos retirados ao redor da planta e dois ramos no topo da planta. Por se tratar de uma cultura de clima temperado, nem todas as partes da planta tiveram o mesmo número de coleta (Tabela 2).

As amostras foram encaminhadas para o Laboratório de Solos e Nutrição de Plantas, da Universidade Estadual de Londrina, onde os frutos, as folhas e os ramos foram pesados em balança semianalítica para a determinação da massa fresca. A medição do comprimento do ramo foi realizada com uma régua graduada. Em seguida, as amostras foram lavadas com água destilada e deionizada para a retirada de possíveis impurezas na superfície das amostras. Os frutos cortados em quatro partes, as folhas e os ramos, separadamente, foram colocados para secar em estufa de circulação forçada de ar, a $55^{\circ} \mathrm{C}$, até atingirem massa constante. Depois de secas, as amostras foram pesadas para a determinação da matéria seca (MS), e moídas em moinho do tipo Willey, com peneira de abertura de $2 \mathrm{~mm}$.

A determinação do nitrogênio nas amostras retiradas foi realizada segundo metodologia descrita em Malavolta et al. (1997). Os dados obtidos foram submetidos ao teste de Tukey, ao nível de 5\% de significância.

\section{RESULTADOS E DISCUSSÃO}

\section{Teor de nitrogênio na folha}

Na Figura 1, verifica-se que, nas épocas de amostragens 1 e 2, não foram observadas diferenças nos teores de $\mathrm{N}$, entre os tratamentos, por serem materiais oriundos de plantas que tiveram a mesma adubação, sendo possível observar diminuição nos teores foliares de $\mathrm{N}$ avaliados entre as épocas 1 e 2 , demonstrando que, possivelmente, houve translocação do $\mathrm{N}$ da folha para outros órgãos (Figura 1).

Na $3^{\text {a }}$ amostragem, o tratamento IP já havia sido adubado no dia 01-07-2006, e apresentou teores superiores aos outros tratamentos, independentemente da dose.

A maior concentração de $\mathrm{N}$ foliar observado em todos os tratamentos foi na $3^{\mathrm{a}}$ amostragem. Para Millard (1996), parte do N estocado nos caules e nas raízes, durante o inverno, é translocado via xilema para a folha. Esse comportamento foi observado em caquizeiro, na Itália, por Bellini (2006), para quem a maior demanda de $\mathrm{N}$ ocorre na primavera e no verão, com consumo de 68\% nessas estações. Essa mesma demanda de $\mathrm{N}$ na estação da primavera foi relatada também por Tagliavini et al. (1998) e O’kennedy et al. (1975), na cultura da macieira. Segundo Neilsen et al. (1997), 80\% do nitrogênio remobilizado na macieira são usados para o crescimento das folhas. Os teores de $\mathrm{N}$ apresentados pelo tratamento IP de 27,70 $\mathrm{g} \mathrm{kg}^{-1} \mathrm{MS}^{-1}$, nessa época de amostragem, estão na faixa dos teores encontrados por Sato et al. (1954) que, trabalhando com caquizeiro no Japão, obtiveram valores de $\mathrm{N}$ foliar variando entre 22,2 e $32,5 \mathrm{~g} \mathrm{~kg}^{-1} \mathrm{MS}^{-1} \mathrm{e}$ próximo dos teores encontrado em folhas de caquizeiro, na Nova Zelândia, por Clark e Smith (1990), de 14,5-26,1 $\mathrm{g} \mathrm{kg}^{-1} \mathrm{MS}^{-1}$.

A partir da $3^{\text {a }}$ amostragem, todos os tratamentos apresentaram declínio dos teores de $\mathrm{N}$, provavelmente, em consequência da redistribuição do $\mathrm{N}$, que aconteceu ao longo dos estádios de desenvolvimento da cultura. Esse comportamento foi observado por Clark \& Smith (1986), no caquizeiro cultivar "Fuyu", onde o teor de $\mathrm{N}$ na folha diminuiu progressivamente ao longo da estação de crescimento da folha, que é mais intenso do que a absorção dos nutrientes. Clark \& Smith (1990), ao efeito da diluição que ocorre nas folhas em crescimento e redistribuição do nutriente para outros órgãos da planta até o final do ciclo.

$\mathrm{Na} 4^{\mathrm{a}}$ amostragem, além do tratamento IP, o tratamento FF já tinha sido aplicado, fato que ocorreu no dia 08-11-06; os dois tratamentos mostraram teores muito próximos e superiores aos outros tratamentos. Todos os tratamentos apresentaram diminuição nos teores de $\mathrm{N}$ em relação à $3^{\mathrm{a}}$ amostragem, tendência que se manteve até o final do ciclo (Figura 1).

Nas folhas amostradas na $5^{\text {a }}$ época, três dias após a $4^{\text {a }}$ aplicação de $\mathrm{N}$, supõe-se que não houve tempo para efeito da aplicação do N. Os tratamentos IP e FF na dose de $160 \mathrm{~kg}$ N/ha e IP na dose de $80 \mathrm{~kg}$ N/ha apresentaram teores superiores aos outros tratamentos de 15-12-06 e da testemunha.

$\mathrm{Na} 6^{\mathrm{a}}$ amostragem, todos os tratamentos resultaram em teores superiores ao tratamento MF e a testemunha. $\mathrm{O}$ baixo teor apresentado pela MF em relação aos outros tratamentos é devido à falta de aplicação de N.

$\mathrm{Na} 7^{\mathrm{a}}$ amostragem de folha, os tratamentos MF e IP, na dose de $80 \mathrm{~kg}$ N/ha, e MF, IP e FF, na dose de $160 \mathrm{~kg}$ N/ha, tiveram os teores de N superiores à testemunha. Esse resultado está de acordo com as observações realizadas por Choi et al. (2003), que encontraram o teor de $\mathrm{N}$ nas folhas do caquizeiro maior quando a adubação com $\mathrm{N}$ foi realizada na 
estação do outono, do que no inverno.

\section{Teor de nitrogênio no ramo}

Na Figura 2, encontram-se os teores de nitrogênio nos ramos frutíferos em função das épocas de amostragem e doses de $\mathrm{N}$ aplicadas.

As amostragens 1 e 2 referem-se aos ramos produzidos no ciclo de 2005-2006, e os demais ao ciclo de 2006-2007. Verifica-se que, nas amostragens 1, 2, 3 e 4, não foram observadas diferenças nos teores de $\mathrm{N}$ entre os tratamentos. Foi observada diminuição nos teores de $\mathrm{N}$ da $1^{\mathrm{a}}$ para a $2^{\mathrm{a}}$ amostragem, em ambas as doses, significando que ainda estava em curso a transferência de fotossintetatos dos ramos e das raízes para outros órgãos.

Os teores de $\mathrm{N}$ encontrados na amostragem 3 foram superiores aos da época de amostragem 2.Esse resultado é consequência da translocação de parte do $\mathrm{N}$ das folhas que estavam próximos da fase de senescência para o ramo, como pode ser observado (Figura 3).

$\mathrm{Na} 4^{\mathrm{a}}$ amostragem, todos os tratamentos, incluindo o IP, apresentaram teores semelhantes, independentemente da dose de $\mathrm{N}$ aplicada. $\mathrm{O}$ tratamento IP não apresentou resposta à aplicação de N.Esse resultado possivelmente ocorreu em consequência do pouco tempo entre a aplicação e a amostragem dos ramos que se encontravam em crescimento.

A amostragem realizada na 5 a época, no tratamento IP com a dose de $160 \mathrm{~kg}$ N/ha, apresentou teor de $\mathrm{N}$ superior à testemunha e ao tratamento de 15-12-2006, na dose de $80 \mathrm{~kg}$ N/ha. A maior absorção de $\mathrm{N}$ apresentada por todos os tratamentos, nesta época de amostragem, ocorreu porque, na estação da primavera, a translocação de $\mathrm{N}$ via xilema é mais intensa em consequência do início da fase de crescimento das folhas. Segundo Millard (1996), o N usado para o crescimento das folhas e inflorescência é translocado via xilema na primavera.

$\mathrm{Na} 6^{\mathrm{a}}$ amostragem, todos os tratamentos apresentaram redução nos teores de $\mathrm{N}$ dos ramos em relação à avaliação anterior. Esse comportamento, provavelmente, indica que o $\mathrm{N}$ presente no ramo foi translocado para os frutos que se encontravam no início de crescimento (Figura 3). O tratamento IP, na dose de $160 \mathrm{~kg}$ N/ha, apresentou teores de $\mathrm{N}$ superiores aos demais tratamentos.

Nos ramos da $7^{\mathrm{a}}$ amostragem, o tratamento de 15-12-06 já havia recebido $\mathrm{N}$, não foi possível verificar aumento no teor de $\mathrm{N}$ nessa amostragem em função do pouco tempo em que a aplicação tenha sido realizada, próximo da colheita. O tratamento IP foi o tratamento que resultou em um teor de $\mathrm{N}$ superior à testemunha, na dose de 80 e $160 \mathrm{~kg} \mathrm{~N} / \mathrm{ha}$.

$\mathrm{Na} 8^{\mathrm{a}}$ amostragem, o tratamento IP, na dose de $160 \mathrm{~kg} \mathrm{~N} / \mathrm{ha}$, apresentou teor maior que os demais tratamentos.

Nos ramos amostrados na $9^{a}$ época, todos os tratamentos apresentaram teores de $\mathrm{N}$ semelhantes entre si, mas ligeiramente superiores àqueles observados na amostragem anterior. Esse comportamento foi observado, também, na $10^{\mathrm{a}}$ amostragem e pode ser atribuído à transferência do $\mathrm{N}$ das folhas que estavam iniciando a senescência e, portanto, próximo da queda das mesmas (Figura 3). Conforme relato de Millard (1996), a translocação de $\mathrm{N}$ das folhas para os órgãos de reserva, em árvores temperadas, varia numa taxa de 20 a 80\%. Titus \& Kang (1982) observaram, também, que as folhas de macieira transferiram o $\mathrm{N}$ para os ramos, quando se aproximavam da senescência. $\mathrm{Na}$ cultura do pessegueiro, Niederholzer et al. (2001) estimaram que aproximadamente $30 \mathrm{~kg}$ de $\mathrm{N}$ por ano são remobilizados das folhas para os ramos durante a senescência das folhas.

Todos os tratamentos apresentaram comprimento de ramo na faixa de 12 a 16 centímetros, e não foi possível verificar a influência das doses de $\mathrm{N}$ utilizadas nesta variável nem sobre o diâmetro médio do ramo, que ficou em torno de $14 \mathrm{~mm}$. Esse resultado foi visualizado em outros trabalhos que constataram a influência do $\mathrm{N}$ no crescimento dos ramos e na produção do pessegueiro cv. Diamante sob diferentes doses de N (Mattos et al., 1991). Estes autores observaram ainda que o $\mathrm{N}$ afeta diretamente o crescimento dos ramos, e que altas quantidades podem levar ao superbrotamento. Costa et al. (1997) verificaram que, no kiwi cv. 'Hayward', quantidades altas de $\mathrm{N}$ resultaram no aumento do comprimento dos ramos. Na macieira, Dong et al. (2005) observaram que a aplicação de $\mathrm{N}$ aumentou significativamente o comprimento dos ramos. Estudos realizados por Basso \& Suzuki (1992) indicam que o N não influiu no comprimento médio das brotações do ano, na macieira cv. Golden delicious. Esse mesmo comportamento foi verificado na Inglaterra, por Marks \& Andrews (1990), que não obtiveram aumento do comprimento dos ramos de anos de adubação, com doses de até $180 \mathrm{~kg} \mathrm{~N} /$ ha/ano. Portanto, pode-se inferir que os tratamentos aplicados não influenciaram no crescimento dos ramos no período avaliado.

\section{Teor de nitrogênio no fruto}

Na Figura 4, é possível observar os teores de nitrogênio nos frutos de todos os tratamentos em função da época de aplicação de 80 e 160 kg N/ha. 
As épocas de amostragens 1 e 2 referem-se aos frutos produzidos no ciclo de 2005-2006, e os demais no ciclo de 2006-2007. Nessas amostragens, não foram observadas diferenças significativas nos teores de $\mathrm{N}$ entre os tratamentos, indicando ser consequência da adubação realizada antes do início do experimento, idêntico para todas as árvores.

Na $3^{\circ}$ amostragem, os tratamentos IP e FF já haviam sido adubados com N, em 01-07-2006 e 08-11-2006, respectivamente. O tratamento IP resultou em maior teor de $\mathrm{N}$ nos frutos em ambas as doses, diferindo-se significativamente dos demais tratamentos somente para a dose de $160 \mathrm{~kg}$ N/ha. Esse comportamento pode ter ocorrido em função de a aplicação ter sido realizada no dia 01-07-2006, quando as plantas se encontravam em dormência e houve tempo suficiente para a absorção e a translocação do $\mathrm{N}$ aplicado até o fruto. Isto indica que a aplicação de $\mathrm{N}$ no solo promoveu efeito no fruto somente após 4 meses da aplicação. Por outro lado, o tratamento FF, independentemente da e em virtude da aplicação de N ter ocorrido no dia 08-11-2006, apresentou teor de $\mathrm{N}$ semelhante ao tratamento IP na dose de $80 \mathrm{~kg}$ N/ha e inferior a de $160 \mathrm{~kg}$ N/ha e semelhante aos outros tratamentos na avaliação realizada no dia 13-11-2006. Esse comportamento aconteceu, provavelmente, porque não houve tempo suficiente para o acúmulo de $\mathrm{N}$ no fruto, sendo que logo em seguida ocorreu o início de crescimento do fruto e o processo de diluição de nutrientes. A maior demanda de $\mathrm{N}$ apresentada por todas as plantas devese à translocação de $\mathrm{N}$ de todos os órgãos da planta para os frutos, que se encontravam no estádio inicial de desenvolvimento (Figura 4).

Para os frutos amostrados na $4^{\mathrm{a}}$ amostragem, o tratamento IP apresentou o maior teor de $\mathrm{N}$ em relação aos demais tratamentos, entretanto o IP $80 \mathrm{~kg}$ $\mathrm{N} /$ ha não diferiu do tratamento FF na mesma dose. Não foi possível observar aumento do teor de $\mathrm{N}$ no tratamento de 15-12-06 em virtude do pouco tempo entre a aplicação e a colheita.

Na $5^{\mathrm{a}}$ amostragem, na dose de $80 \mathrm{~kg} \mathrm{~N} / \mathrm{ha}$, observa-se que o tratamento IP resultou no maior teor de $\mathrm{N}$ dos frutos $\left(5,57 \mathrm{~g}\right.$ de $\left.\mathrm{N} \mathrm{kg}^{-1} \mathrm{MS}^{-1}\right)$, diferindo do tratamento FF, que foi de 4,37 $\mathrm{g}^{\mathrm{de}} \mathrm{N} \mathrm{kg}^{-1} \mathrm{MS}^{-1}$, e dos demais tratamentos.

Para a $6^{\mathrm{a}}$ amostragem, o tratamento IP $80 \mathrm{~kg}$ $\mathrm{N} /$ ha apresentou maior teor de $\mathrm{N}$ em relação aos outros tratamentos, mas diferiu apenas dos tratamentostestemunha, MF e FF, na dose de $80 \mathrm{~kg}$ N/ha.

Os resultados obtidos neste experimento contrariam as observações feitas em outros trabalhos com árvores de clima temperado, isto é, que o teor de $\mathrm{N}$ no fruto tende a diminuir quando se aproxima a colheita. Segundo Huett \& Stewart (1999) constataram, a demanda de $\mathrm{N}$ no pessegueiro diminui quando se aproxima a maturação dos frutos. Já Hanson \& Howell (1995) reportaram que, na videira, a maior demanda de $\mathrm{N}$ ocorre do florescimento até a mudança de cor dos frutos e que, depois desse estádio, o teor de $\mathrm{N}$ tende a diminuir até a colheita.

TABELA 1 - Resultados das análises químicas das amostras de solo das camadas de 0-20 e 20-40 cm.

\begin{tabular}{|c|c|c|c|c|c|c|c|c|c|c|}
\hline Camada & $\mathrm{pH}$ & P(Melhlich) & $\mathrm{Ca}^{+2}$ & $\mathrm{Mg}^{+2}$ & $\mathrm{~K}^{+}$ & $\mathrm{Al}^{+3}$ & $\mathrm{H}+\mathrm{Al}$ & CTC & $\mathrm{V}$ & M.O. \\
\hline$(\mathrm{cm})$ & $\mathrm{CaCl}_{2}$ & $\mathrm{mg} \mathrm{dm}^{-3}$ & & .......... & $\mathrm{mol}_{\mathrm{c}}$ & & & &.$\%$ & $\mathrm{~g} \mathrm{dm}^{-3}$ \\
\hline $0-20$ & 5,15 & 18,3 & 2,9 & 1,1 & 0,18 & 0,15 & 4,01 & 8,19 & 51 & 13,2 \\
\hline $20-40$ & 5,04 & 9,0 & 2,7 & 1,2 & 0,19 & 0,05 & 5,35 & 9,43 & 43 & 15,9 \\
\hline
\end{tabular}

M.O. - Matéria Orgânica Walkle Black.

TABELA 2- Datas das amostragens das folhas, frutos.

\begin{tabular}{|c|c|c|c|c|c|c|c|c|c|c|}
\hline \multirow{4}{*}{$\begin{array}{c}\text { Órgãos } \\
\text { da planta } \\
\text { Folha }\end{array}$} & \multicolumn{10}{|c|}{ Épocas de amostragens } \\
\hline & \multicolumn{7}{|c|}{ Ano de 2006} & \multicolumn{3}{|c|}{ Ano de 2007} \\
\hline & $1^{\mathrm{o}}$ & $2^{\circ}$ & & & $3^{\circ}$ & $4^{\circ}$ & $5^{\circ}$ & $6^{\circ}$ & $7^{\circ}$ & \\
\hline & $02 / 04$ & $17 / 05$ & --- & & 29/09 & $13 / 11$ & $18 / 12$ & $01 / 02$ & $18 / 03$ & --- \\
\hline \multirow{2}{*}{ Ramo } & $1^{\mathrm{o}}$ & $2^{\circ}$ & $3^{\circ}$ & $4^{\circ}$ & $5^{\circ}$ & $6^{\circ}$ & $7^{\circ}$ & $8^{\circ}$ & $9^{\circ}$ & $10^{\circ}$ \\
\hline & $02 / 04$ & $17 / 05$ & 01/07 & $15 / 08$ & 29/09 & $13 / 11$ & $18 / 12$ & $01 / 02$ & $18 / 03$ & $02 / 05$ \\
\hline \multirow{2}{*}{ Fruto } & $1^{\mathrm{o}}$ & $2^{\circ}$ & & \multirow{2}{*}{---} & & $3^{\circ}$ & $4^{\circ}$ & $5^{\circ}$ & $6^{\circ}$ & \\
\hline & $02 / 04$ & $17 / 05$ & --- & & --- & $13 / 11$ & $18 / 12$ & 01/02 & $18 / 03$ & --- \\
\hline
\end{tabular}

---- Não houve amostragem. 


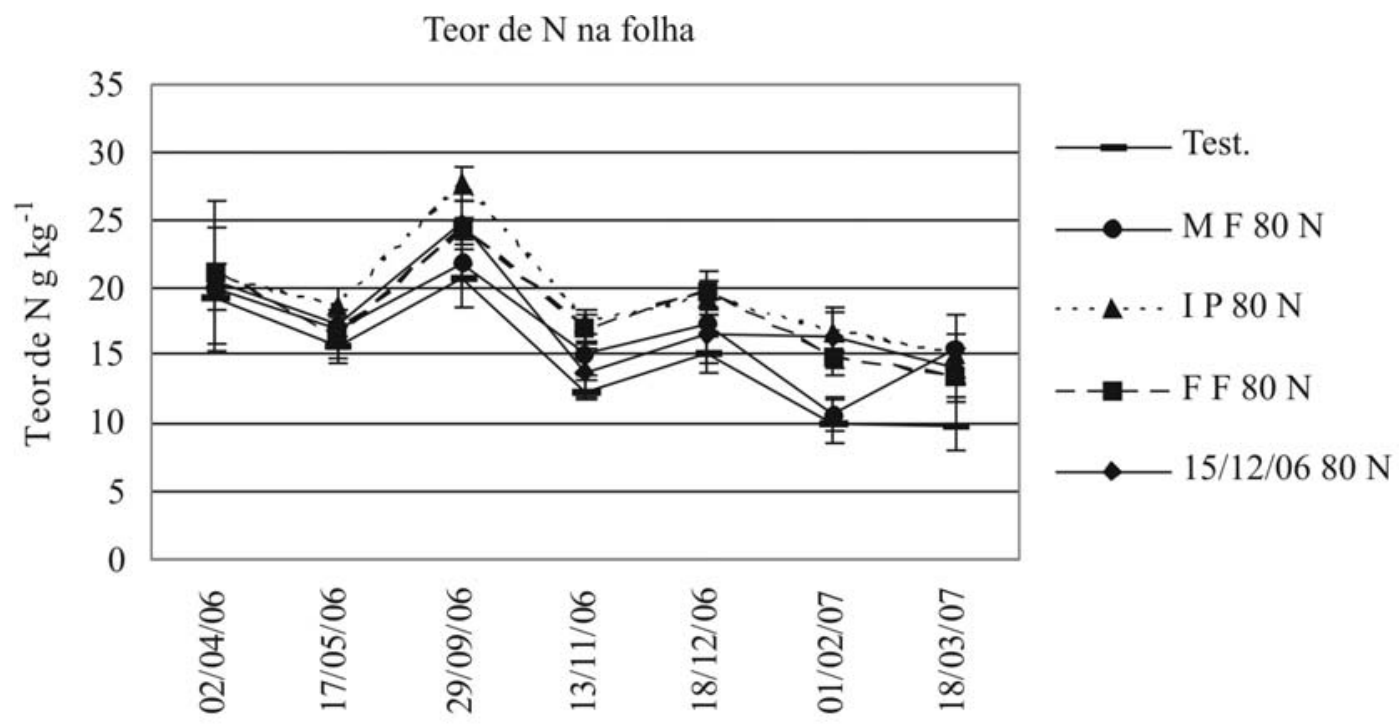

Épocas de coletas

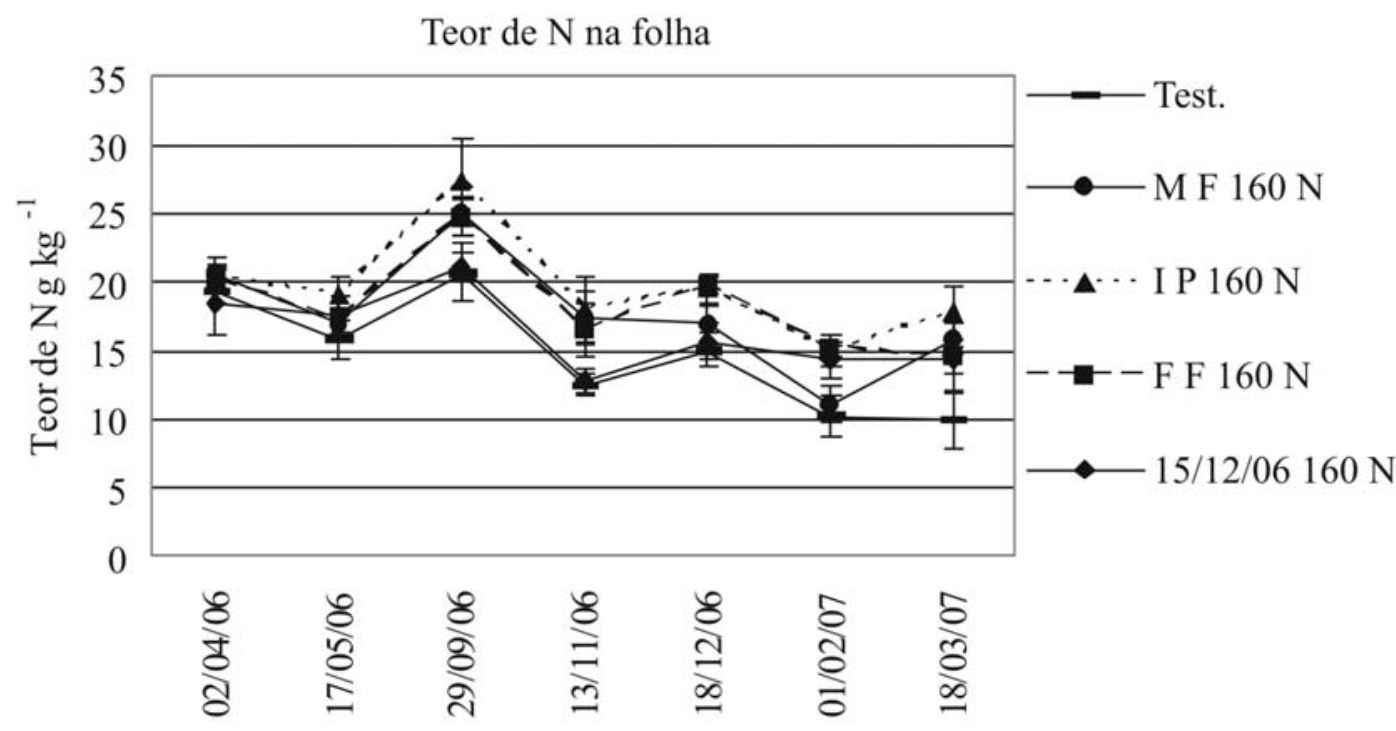

Épocas de coletas

FIGURA 1-Teor de $\mathrm{N}$ na folha ao longo do estádio de desenvolvimento da cultura em função da dose de 80 e $160 \mathrm{~kg}$ de N/ha. 


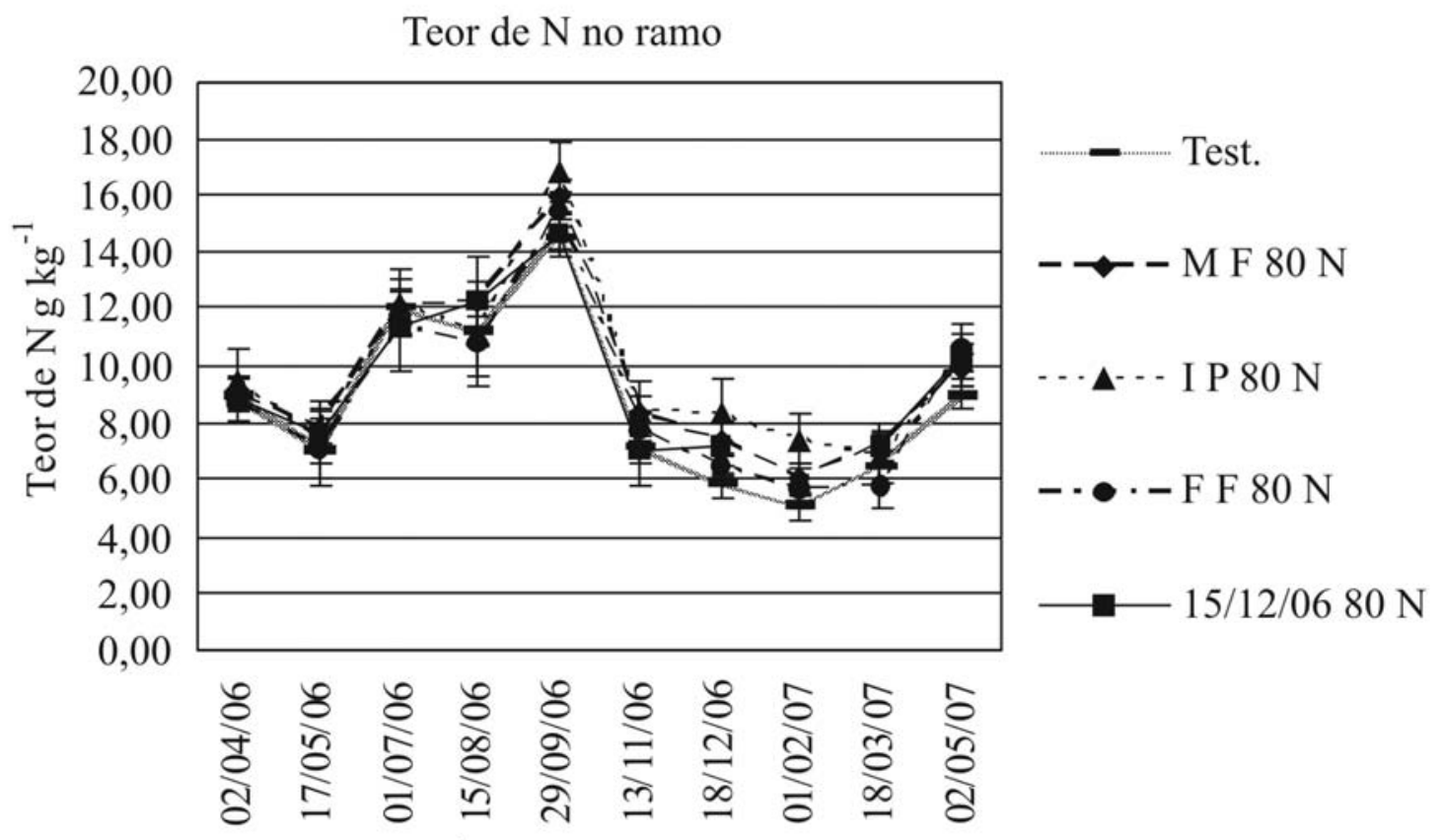

Épocas de coletas

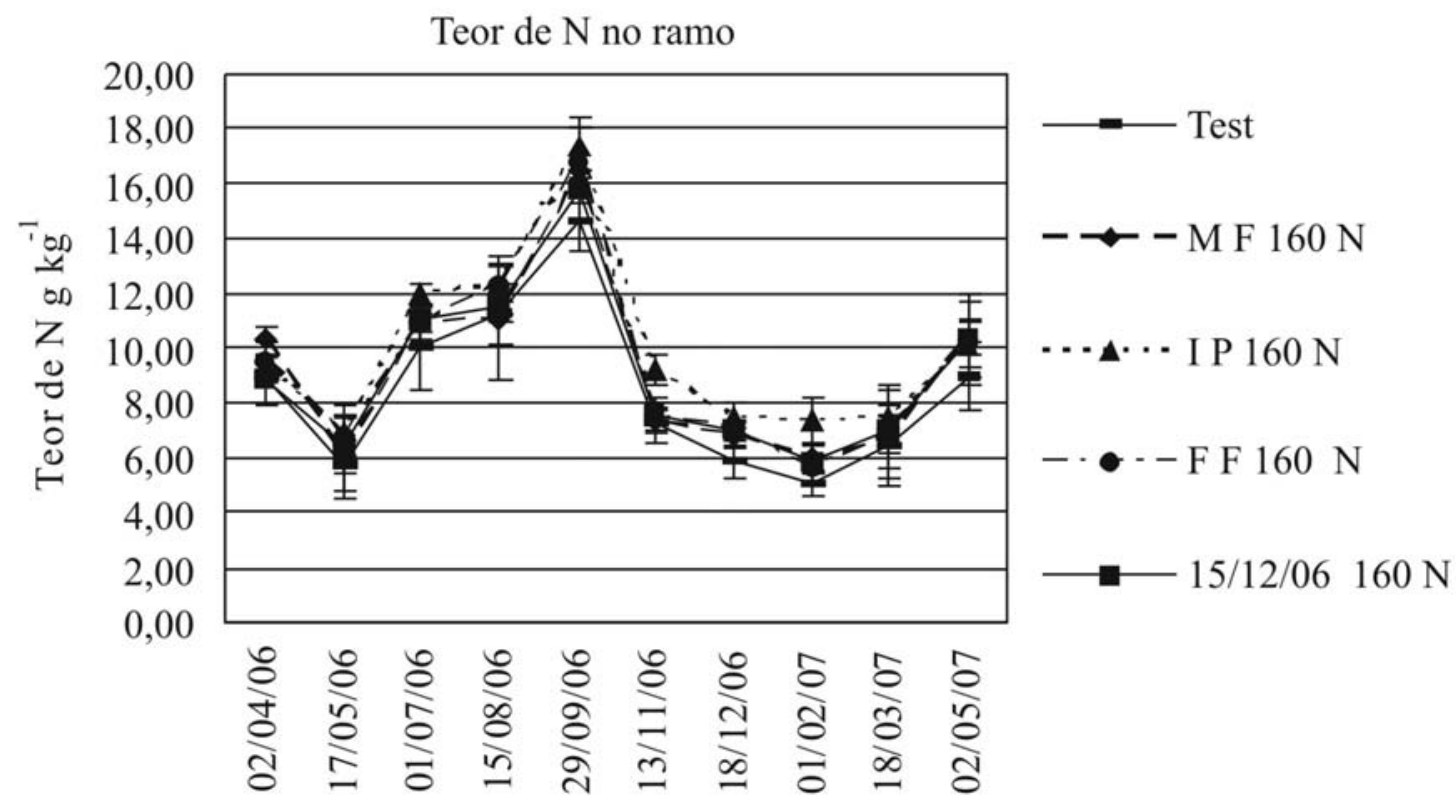

Épocas de coletas

FIGURA 2- Teor de N no ramo ao longo do estádio de desenvolvimento da cultura em função da dose de 80 e $160 \mathrm{~kg}$ de N/ha. 


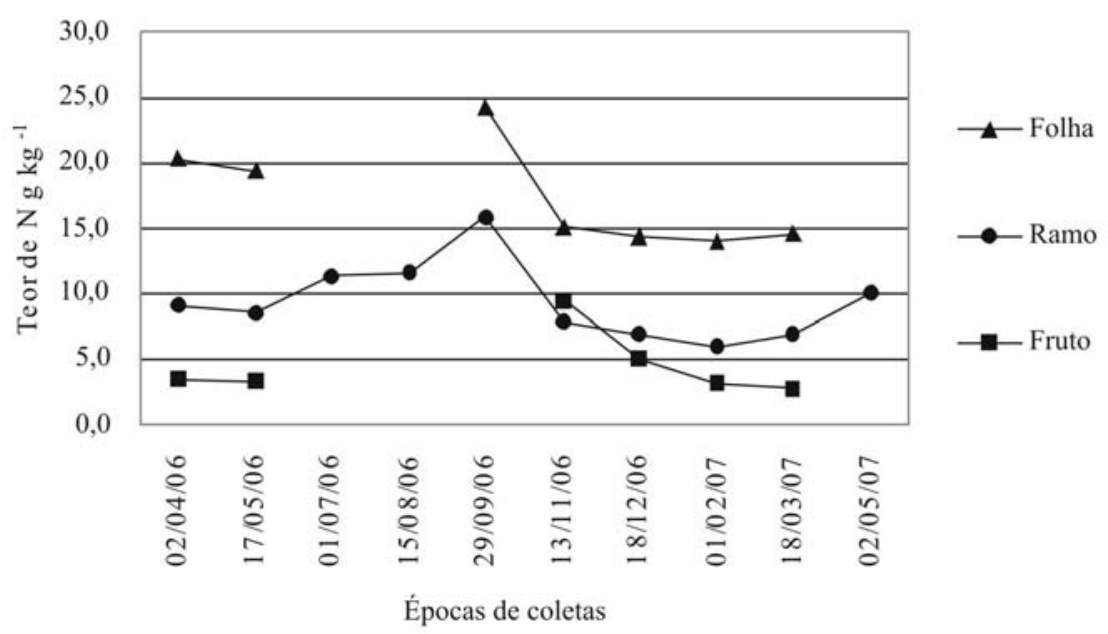

FIGURA 3- Teor médio de N nas folhas, ramos e frutos, em função da época de amostragem (Média de todos os tratamentos para cada órgão).
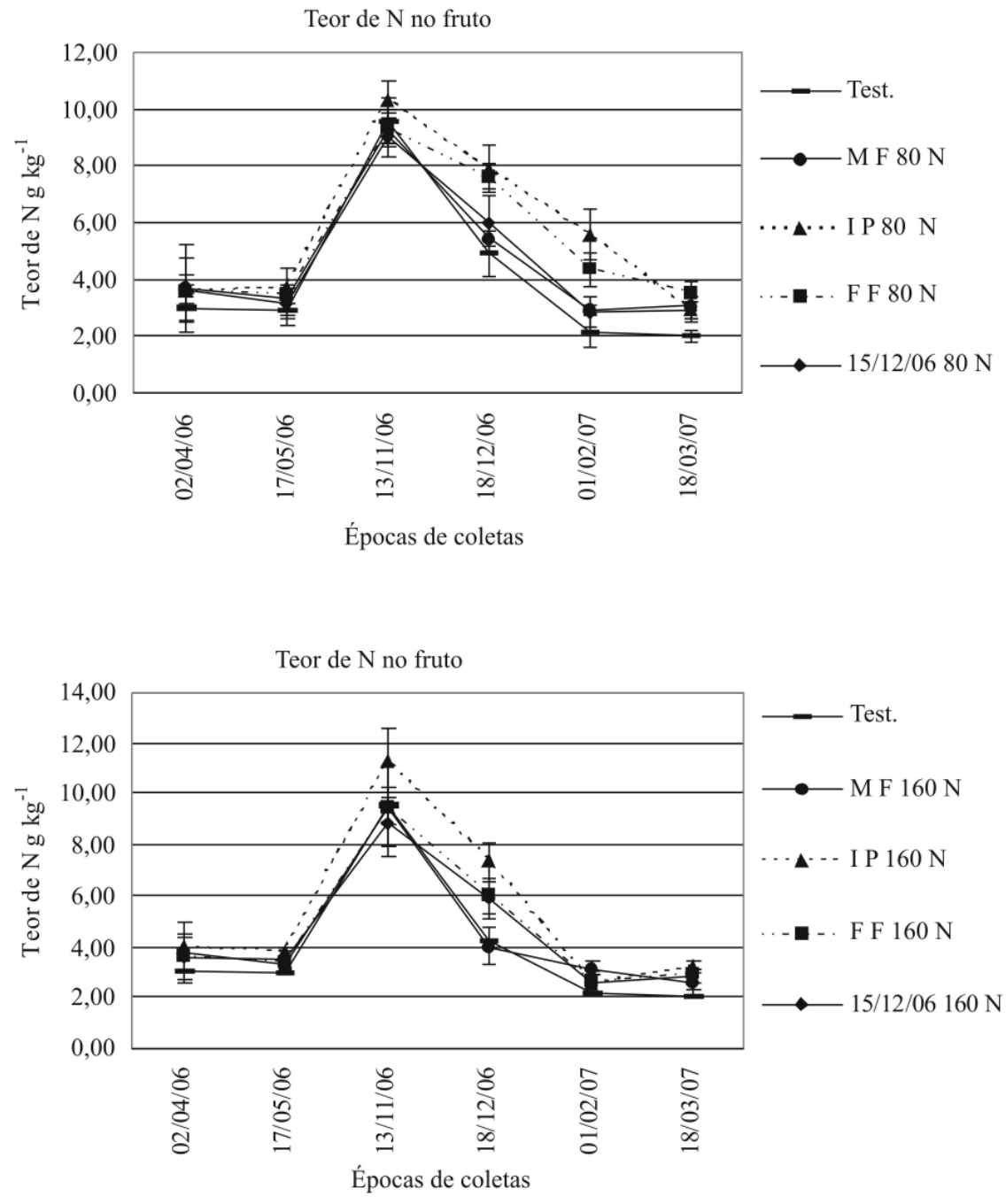

FIGURA 4 -Teor de nitrogênio no fruto em função de épocas de amostragem e doses de N 80 kg/ha e $160 \mathrm{~kg} / \mathrm{ha}$. 


\section{CONCLUSÕES}

1-Independentemente do tratamento, a concentração de $\mathrm{N}$ foi maior em todos os órgãos no início do ciclo.

2-No fruto, o tratamento no início de poda apresentou maior teor de $\mathrm{N}$ na primavera, em ambas as doses aplicadas, enquanto no ramo este tratamento apresentou maior teor de $\mathrm{N}$ no verão.

3-Na folha, os tratamentos IP e MF apresentaram os maiores teores de $\mathrm{N}$ na primavera e no verão, e diminuição significativa na fase final do ciclo, antes da senescência e queda

4-Na folha e no ramo, todos os tratamentos resultaram em teor de $\mathrm{N}$ semelhante no final do ciclo.

5-A época de aplicação no início de poda foi a que demonstrou menor variação de $\mathrm{N}$ independente da dose.

\section{REFERÊNCIAS}

BELLINI, E. Cultural practices for persimmon production. Zaragoza : CIHEAM-IAMZ, 2002. p. 39-52. Disponível em: <http://ressources.cihea. org/om/pdf/a51/02600061.pdf> Acesso em: 10 fev. 2006.

CLARK, C. J.; SMITH, G. S. Leaf analysis of persimmons. Growing Today, Auckland, n. 3, v. 4, p. 15-17, 1986.

CLARK, C. J.; SMITH, G. S. Seasonal changes in the mineral nutrient content of persimmon leaves. Scientia Horticulturae, Amsterdam, v. 42, n. 1-2, p. 85-97, 1990.

CHOI, S. T.; PARK, D. S.; SONG, W. D.; KANG, S. M.; SHON, G. M. Effect of different degrees of defoliation on fruit growth and reserve accumulation in young 'fuyu' trees. Acta Horticulturae, Queensland, v. 601, p. 99-104, 2003.

COSTA, G.; Lain, O.; VIZZOTTO, G.; JOHNSON $S$. Effect of nitrogen fertilization on fruiting and vegetative performance, fruit quality and post-harvest life of Kiwifruit cv Hayward. Acta Horticulturae, Wageningen, v.444, p.279-284, 1997.
DONG, S. F.; CHENG, L. L.; SCAGEN, C. F.; FUCHIGANI, H. Method of nitrogen application in summer affects plant growth and nitrogen uptake in autumn in young Fuji/ M26 apple trees. Communication in soil science and plant analysis, New York, v. 36, n. 11-12, p. 1465-1477, 2005.

EMBRAPA - Centro acional de Pesquisa de Solos. Sistema brasileiro de classificação de solos. Brasília, 2006. 412p.

EPSTEIN, E. Nutrição mineral de plantas: princípios e perspectivas. São Paulo: Rio de Janeiro: Livros Técnicos e Científicos, 2005. 341p.

ERNANI, P. R.; DIAS, J.; BORGES, M. A aplicação de nitrogênio ao solo em diferentes estádios não afetou o rendimento de frutos de cultivares de macieira. Ciência Rural, Santa Maria, v. 30, n. 2, p. 223-227, 2000.

GASANOV, Z. M. Nitrogen nutrition and productivity of persimmon. Horticultural Abstracts, n. 55, v. 5, p 39, 1984.

GEORGE, A. P.; NISSEN, R. J.; BROADLEY, R. H.; COLLINS, R. J. Improving the nutritional management of non-astringent persimmon in subtropical australia. Acta Horticulturae, Queensland, v. 1, n. 34, p. 131-138, Mar. 2003.

HANSON, E.J.; HOWELL, S. Nitrogen accumulation and fertilizer use efficiency by grapevines in short-season growing areas. HortScience, Alexandria, v. 30, n. 3, p. 504-507, 1995.

HUETT, D. O.; STEWART, G. R. Timing of $15 \mathrm{~N}$ fertiliser application, partitioning to reproductive and vegetative tissue, and nutrient removal by fieldgrown low-chill peaches in the subtropics. Australian Journal of Agricultural, Victoria, v.50, p.211-215, 1999.

IBGE - Instituto Brasileiro de Geografia e Estatística. Cidades@. Disponível em: <http://www.ibge.gov. br/cidadesat/default.php> Acesso em: 18 jun. 2009.

MALAVOLTA, E.; VITTI, G. C.; OLIVEIRA, S. A. Avaliação do estado nutricional das plantas. Piracicaba: Potafós, 1997. 
MARKS, M. J.; ANDREWS, L. The response of bramley's seedling apple trees grown on different rootstocks to spring and autumn aplliend nitrogen. Acta Horticulturae, Warsaw, v. 273, p. 321-329, 1990.

MARTINS, F. P.; PEREIRA, F. M. Cultura do caquizeiro. Jaboticabal: Funep, 1989. 71p.

MARSCHNER, H. Mineral nutrition of higher plants. San Diego: Academic Press, 1995.

MATTOS, M. L. T.; FREIRE, C. J. S.; MAGNANI, M. Produção do pessegueiro cv. Diamante, sob diferentes doses de nitrogênio aplicado ao solo. Pesquisa Agropecuária Brasileira, Brasília, v. 26, n. 1, p. 113-117, 1991.

MILLARD, P. Ecophysiology of the internal of nitrogen for tree growth. Jounal of Plant Nutrition and Soil Science, Hoboken, v. 159, p 1-10, 1996.

MUÑOZ, N.; GUERRI, J.; LEGAZ, F.; PRIMO, M. E. Seasonal uptake of $15 \mathrm{~N}-$ nitrate and distribution of absorbed nitrogen in peach trees. Plant Soil, Dordrecht, v.150, p.263-269, 1993.

NIEDERHOLZER, F. J. A.; DEJONG, T. M.; SAENZ, J. L.; MURAOKA, T. T. ; WEINBAUM, S. A. Effectiveness of fall vs. spring fertilization of field grown peach trees . Journal of the American Society for Horticultural Science, Alexandria, v. 126, n. 5, p. 644-648, 2001.

NEILSE, D.; MILLARD, P.; NEILSEN, G. H.; HOUGUE, E. J. Sources of N used for leaf growth in a high density apple (Malus domestica) orchard irrigated with ammonium nitrate solution. Tree physiology, Victoria, v. 17, p. 733-739, 1997.
O’KENNEDY, B. T.; HENNERTY, M. J.; TITUS, J. $S$. The effects of autumn foliar urea sprays on storage forms of nitrogen extracted from bark and wood of apple shoots. Journal of Horticultural Science, Alexandria, v.50, p.331-338, 1975.

PARK, K. J.; TUBONI, C. T.; OLIVEIRA, R. A.; PARK, K. J. B. Estudo da secagem de caqui giombo com encolhimento e sem encolhimento. Revista Brasileira de Produtos Agroindustriais, Campina Grande, v. 6, n. 1, p. 71-86, 2004.

SATO, K.; ISHIHARA, M.; HARADA, R. Studies on leaf analyse of fruit trees Leaf analysis in Japanese persimmon orchard. Bulletin of the National Institute of Agricultural Sciences, Tokyo, Series E., v. 3, p. 169-186, 1954.

TAGLIAVINI, M.; MILLARD, P.; QUARTIERI, M. Storage of foliar-absorbed nitrogen and remobilization for spring growth in young nectarine (Prunus persica var. nectarina) trees. Tree Physiology, Victoria, v. 18, n. 3, p. 203-207, 1998.

TITUS, J. S.; KANG, S. Nitrogen metabolism, translocation and recycling in apple trees . Horticultural Reviews, New York, v.4, p.204-246, 1982.

VIGLIONE, J.H.; KIIHL, L.R.B.; CARAMORI, P.H.; OLIVEIRA, D. Cartas climáticas do Paraná. Londrina: IAPAR, 2000. CD-ROM 